\title{
Intramyocardial Transplantation of Umbilical Cord Mesenchymal Stromal Cells in Chronic Ischemic Cardiomyopathy: A Controlled, Randomized Clinical Trial (HUC-HEART Trial)
}

\author{
A. Tulga Ulus ${ }^{1}$, Ceren Mungan ${ }^{2}$, Murat Kurtoglu $^{3}$, Ferda Topal Celikkan ${ }^{4}$, Mesut Akyol ${ }^{5}$, \\ Merve Sucu ${ }^{2}$, Mustafa Toru ${ }^{6}$, Serdar Savas $\mathrm{Gul}^{7}$, Ozgur Cinar ${ }^{4}$, Alp Can ${ }^{4}$ \\ ${ }^{1}$ Department of Cardiovascular Surgery, Hacettepe University Faculty of Medicine, Ankara, Turkey \\ ${ }^{2}$ Ankara University Biotechnology Institute and Sisbiyotek, Ankara, Turkey \\ ${ }^{3}$ Cardiovascular Surgery Division, Ankara Guven Hospital, Ankara, Turkey \\ ${ }^{4}$ Department of Histology and Embryology, Laboratory for Stem Cells and Reproductive Cell Biology, Ankara University School of \\ Medicine, Ankara, Turkey \\ ${ }^{5}$ Department of Biostatistics, Ankara Yildirim Beyazit University, Ankara, Turkey \\ ${ }^{6}$ Radiology Division, Ankara Liv Hospital, Ankara, Turkey \\ ${ }^{7}$ Visart Medical Imaging Center, Ankara, Turkey
}

Background and Objectives: The HUC-HEART Trial (ClinicalTrials.gov Identifier: NCT02323477) was a controlled, prospective, phase I/II, multicenter, single-blind, three-arm randomized study of intramyocardial delivery of human umbilical cord-derived mesenchymal stromal cells (HUC-MSCs) combined with coronary artery bypass-grafting (CABG) in patients with chronic ischemic cardiomyopathy (CIC). The trial aimed to assess (i) the safety and the efficacy of cell transplantation during one-year follow-up, (ii) to compare the efficacy of HUC-MSCs with autologous bone-marrow-derived mononuclear cells (BM-MNCs) in the same clinical settings.

Methods and Results: Fifty-four patients who were randomized to receive HUC-MSCs $\left(23 \times 10^{6}\right)(\mathrm{n}=26)$ or BM-MNCs $\left(70 \times 10^{7}\right)(n=12)$ in combination with CABG surgery. The control patients $(n=16)$ received no cells/vehicles but CABG intervention. All patients were screened at baseline and 1, 3, 6, 12 months after transplantation. Forty-six (85\%) patients completed 12 months follow-up. No short/mid-term adverse events were encountered. Decline in NT-proBNP (baseline 6 months) in both cell-treated groups; an increase in left ventricular ejection fraction (LVEF) (5.4\%) and stroke volume (19.7\%) were noted (baseline 6 or 12 months) only in the HUC-MSC group. Decreases were also detected in necrotic myocardium as $2.3 \%$ in the control, $4.5 \%$ in BM-MNC, and $7.7 \%$ in the HUC-MSC groups. The 6-min walking test revealed an increase in the control (14.4\%) and HUC-MSC (23.1\%) groups.

Conclusions: Significant findings directly related to the intramyocardial delivery of HUC-MSCs justified their efficacy in CIC. Stricter patient selection criteria with precisely aligned cell dose and delivery intervals, rigorous follow-up by detailed diagnostic approaches would further help to clarify the responsiveness to the therapy.

Keywords: Bone marrow MSC, Clinical trial, Ischemic cardiomyopathy, Stem cell therapy, Umbilical cord MSC

Received: May 4, 2020, Revised: June 18, 2020, Accepted: July 6, 2020, Published online: August 31, 2020

Correspondence to Alp Can

Department of Histology and Embryology, Laboratories for Stem Cells and Reproductive Medicine, Ankara University School of Medicine, Sihhiye, Ankara 06410, Turkey

Tel: +903125958169, Fax: +903123103010

E-mail: alpcan@medicine.ankara.edu.tr

(c) This is an open-access article distributed under the terms of the Creative Commons Attribution Non-Commercial License (http://creativecommons.org/licenses/by-nc/4.0/), which permits unrestricted non-commercial use, distribution, and reproduction in any medium, provided the original work is properly cited.

Copyright (C) 2020 by the Korean Society for Stem Cell Research 


\section{Introduction}

Chronic ischemic cardiomyopathy (CIC) with moderate-to-severe left ventricle (LV) systolic dysfunction is frequently associated with scar tissue formation resulting from an acute infarction of the myocardium and loss of cardiomyocytes. The regeneration process of the infarcted myocardium requires to clearing damaged and dysfunctional dead tissue, regulating inflammation, suppressing overactive fibrosis (scar tissue), and reconstituting and integrating healthy cardiomyocytes, extracellular matrix, blood and lymphatic vessels (1). Besides medical and interventional care of CIC, several therapeutic attempts have been performed to improve the clinical conditions of patients by promoting the replacement of the scar tissue and therefore the lost cardiomyocytes and by activating cardiac repair.

Different cell types including skeletal myoblasts, bone marrow-originated stem cells and mesenchymal stromal cells from different tissue origins, cardiac resident cells, and finally, embryonic stem cells have been tested in clinical trials, as listed and briefly reviewed by Ghiroldi et al. (2) demonstrating varying degrees of effectiveness. In general, clinical trials have produced conflicting and inconclusive results using a range of stem cells, and there is still debate regarding the best patient group, source of cells, and the route and timing of administration. Clear-cut evidence on what type of cells should be adopted to CIC is still lacking, because results show significant heterogeneity in terms of efficacy. Most clinical trials used autologous bone marrow mononuclear cells (BM-MNCs), which comprise predominantly mononuclear cells, with small sub-populations of hematopoietic stem cells and mesenchymal stromal cells (MSC) (3, 4). According to two meta-analysis of more than 20 clinical trials on the effectiveness of BM-MNCs for cardiac regeneration following acute myocardial infarction (MI), it is clear that no significant improvement was observed in the mortality and morbidity of patients who received BM-MNCs, although a significant and sustained improvement in left ventricular ejection fraction (LVEF) was reported $(5,6)$. The use of autologous and allogeneic MSCs in various clinical settings has increased in recent years. A number of clinical trials have been conducted to test the effects of MSCs on cardiac repair and regeneration aiming to restore damaged heart muscle tissue, with these cells being credited with displaying stemness properties. Accumulating evidence from those clinical trials supports the notion that ex vivoexpanded MSCs are safe and possess the capacity for repair, making them a potential treatment of chronic heart diseases characterized by fibrotic scar tissue. However, comparing the different trials is a challenging task owing to the vast differences in patient profiles, cell phenotypes, dosing, routes of delivery, study endpoints and design, which ranged from small, open-label protocols lacking true controls and clouded by concomitant procedures such as coronary artery bypass graft (CABG) surgery, to rigorously conducted, randomized, adequately powered, placebo-controlled, blinded designs with outcome measures based on clinically relevant markers.

Human umbilical cord-derived mesenchymal stromal cells (HUC-MSCs), considered as perinatal stem cells, have been of great interest because of their higher capacity of self-renewal compared to adult MSCs, senescence-resistant, and higher angiogenic and paracrine effects because they hold the properties of both embryonic and adult stem cells (7). To date, more than 100 clinical studies have been conducted and finalized regarding the safety and efficacy of HUC-MSC transplantation in publicly important major disorders including cardiac diseases and dysfunctions (8), in which they have shown promising results mainly due to their pro-angiogenic and anti-inflammatory properties. However, no trial has yet been published of direct application of these cells into the infarcted myocardium. Over recent years, the safety and efficacy of HUC-MSCs on a relatively small number of patients with ischemic cardiomyopathy were examined where cells were delivered via intravenous or intracoronary routes (9-16).

This pioneering study, named as the HUC-HEART Trial (ClinicalTrials.gov Identifier: NCT02323477) for short, has been designed as a phase $1 / 2$, controlled, multicenter, single-blind, randomized, three-arm clinical study of the intramyocardial delivery of allogeneic HUC-MSCs in patients with CIC. Patients undergoing CABG but not receiving any cells or vehicle served as the control group. The rationale of using autologous BM-MNCs as an another group originated from the facts that (i) BM-MNCs are one of the most commonly tested cell types among the other stem/progenitor cells that exhibited crucial improvements in ventricular functions including LVEF increase and reduced infarct size (reviewed in $(1,2)$ ); (ii) the relative ease of cell collection by a minimally invasive procedure and requiring minimal manipulation before administration; (iii) being a heterogeneous population of stem cells isolated from the bone marrow. The detailed study schema including inclusion, exclusion criteria and primary, secondary endpoints was previously published elsewhere in 2015 (14). The ultimate aim of this study was to address the issues mentioned above by providing evi- 
dence of the effects of HUC-MSC transplantation in combination with $\mathrm{CABG}$ on ventricular function, as well as on patient clinical outcomes. The preliminary results pertaining to few patients from each group were presented as a poster at a meeting in 2017 (9). Here, we report the results of the overall study, which was finalized in December 2018 when the last patient was evaluated following one-year of follow-up.

\section{Materials and Methods}

\section{Patient selection and study design}

The study design was primarily approved by the Institutional Ethical Review Board (11/25/2013, \#18-704-13); the onset and legislative issues were officially permitted by the Ministry of Health and the Advisory Board of Stem Cell Transplantations (1/30/2014, \#19738). The HUC-HEART Trial enrolled patients between May 2015 and November 2017; the surgical interventions were performed in three hospitals. Informed consent was obtained from all individual participants included in the study. The trial was terminated on December 2018 when reaching the officially-allowed time point and budget limit. For eliminating the confounder factor arising from the sex variation, and because of the higher incidence of male patients with CIC requiring CABG, only male subjects aged between 30 80 years who presented with CIC with an indication for $\mathrm{CABG}$ and decreased global left ventricular ejection fraction (LVEF) as determined via baseline cardiac magnetic resonance imaging (MRI) and echocardiography (Echo) were included. Inclusion and exclusion criteria were listed in Supplementary Table S1. Primary endpoint was assigned as the emergence of major adverse cardiac event rate compared with the control group (defined as the composite incidence of death, hospitalization for worsening heart failure, or nonfatal recurrent myocardial infarction, and ventricular arrhythmia); secondary endpoints were assigned as significant changes in LVEF (time frame: preoperatively, postoperatively (12 months), or in infarcted area. The assignment of patients into groups was implemented by two of the authors, based on the block randomization protocol using the Random Allocation Software version 1.0, and the block randomization method (14).

The following null hypothesis was used in order to calculate the required sample size; patients undergoing HUCMSC injections in association with CABG would not exhibit different $L V E F$ values than those undergoing either BM-MNC administration and CABG or CABG alone, during a 12-month follow-up. Three independent groups of patients were included in the HUC-HEART Trial in a 1 :
$1: 2$ pattern. The sample size and the study power were calculated using computer-based software and published previously (14). Following numbers of patients could complete the total follow-up; the first group served as the control group $(n=15)$; these patients did not undergo cell transplantation. The second group $(n=9)$, which received autologous BM-MNCs, served as the second control group. The third group $(n=22)$ received allogeneic HUC-MSCs (Fig. 1A). All interventions were performed in combination with CABG surgery. MR, SPECT (Single-photon emission computed tomography), and PET (positron emission tomography) imaging were performed by two core facilities. All interventions and imaging analyses were implemented by independent imaging specialists and statisticians who were blinded. The participants were not blinded to their group allocation.

\section{Cells preparation and transfer}

Two different cell isolation procedures were used for the BM-MNCs and HUC-MSCs. Autologous BM-MNC isolation was performed as published (14). The concentrate consisted of a heterogeneous, nucleated cell population including hematopoietic, mesenchymal, other progenitor cells, as well as granulocytes and platelets. A total of approximately $70 \times 10^{7} \mathrm{BM}-\mathrm{MNCs}$ were transplanted to each patient. A small sample from each transplanted batch of cells was subsequently analyzed immunophenotypically including $\mathrm{CD}_{3}{ }^{+}$and $\mathrm{CD} 45^{+}$markers as common BM stem/progenitor cell indicators (Supplementary Table S2).

For patients in group 3, raw umbilical cord sections were collected under aseptic conditions via cesarean sections involving full-term infants following the acquisition of written consent from the mother. The cells used were obtained from the same cell production facility obtained from a single cord donor. All cell isolations, expansions, safety and quality validation procedures, as documented elsewhere (14) were compliant with good manufacturing practice (GMP) guidelines. The final number of transported cells $\left(21 \sim 26 \times 10^{6}\right)$ at $2 \sim 10^{\circ} \mathrm{C}$ (Supplementary Table S2) was diluted in $4 \mathrm{ml}$ Ringer's lactate solution containing $1 \%$ human serum albumin (17).

\section{Surgical procedure, intervention and follow-up}

On the day of cell preparation and delivery, patients underwent conventional CABG surgery with/without cardiopulmonary bypass. After completion of the distal coronary anastomoses, intraoperative injections into 10 periinfarct areas were performed using standardized 27-gauge syringes. To visualize the target areas for the cell injections selected via preoperative MRI analysis, a segmental map 
A

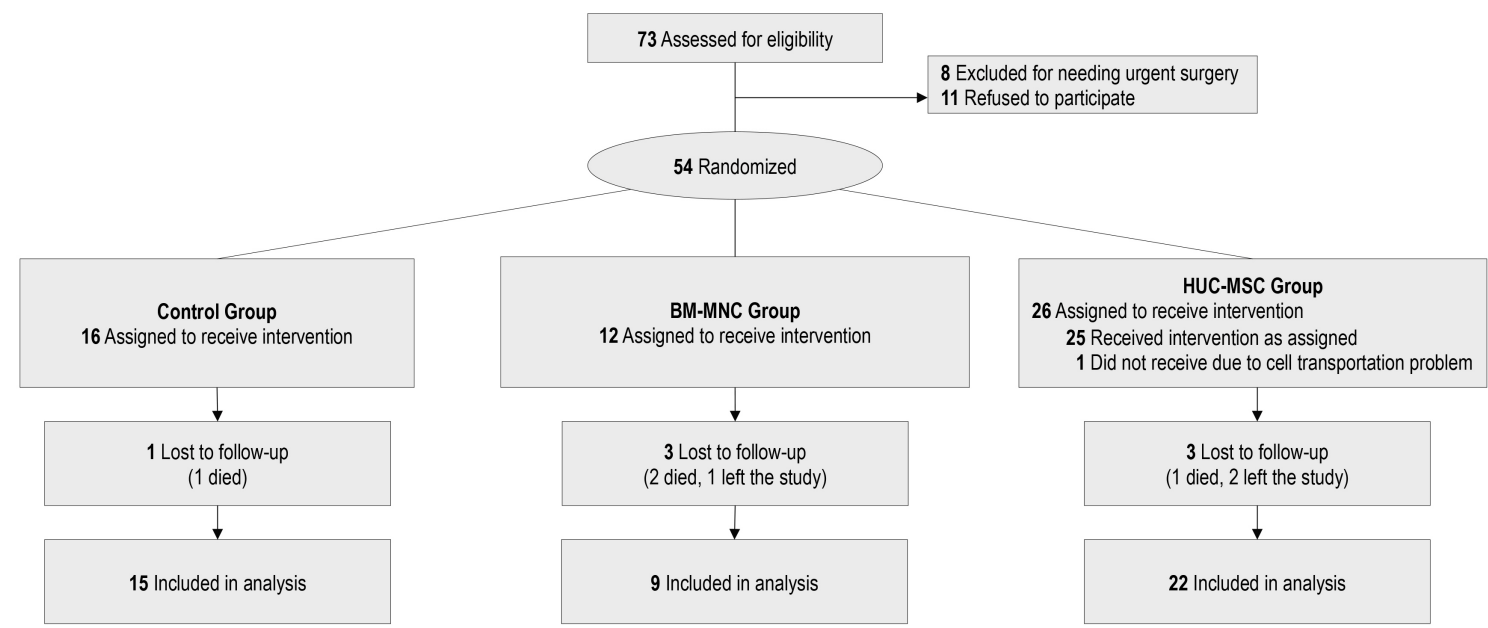

B

Pre-transplantation

Post-transplantation (months)

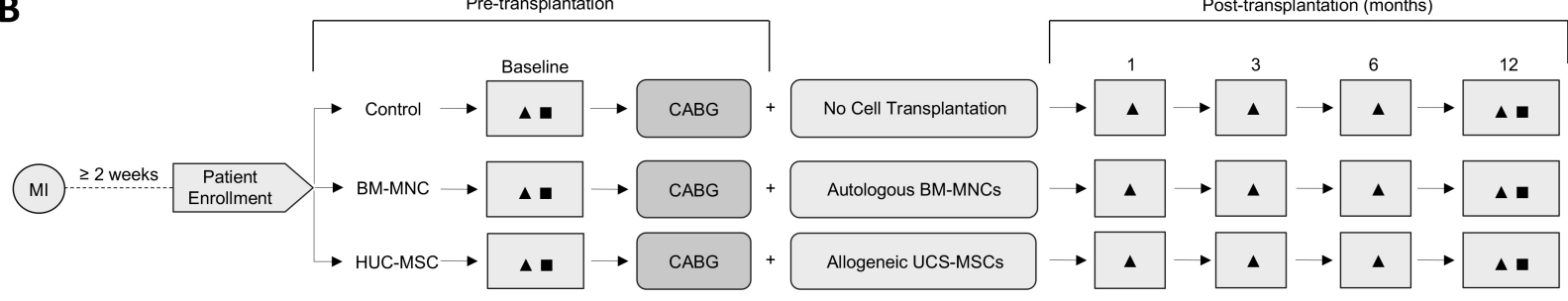

Fig. 1. (A) Subject throughput from enrollment $(n=73)$ to data analysis $(n=46)$. (B) A patient follow-up chart. BM-MNC: bone marrow mononuclear cells, HUC-MSC: human umbilical cord mesenchymal stromal cells, Ml: myocardial infarction, CABG: coronary artery bypass grafting, $\mathbf{\Delta}$ : cardiac evaluation including electrocardiography (ECG); echocardiography (Echo); Holter rhythm monitoring; 6-min walking test; cardiac enzymes (CK-MB, LDH, troponin I); 27-parameter blood tests, including complete blood count; 22-parameters biochemical tests; and NT-proBNP measurements, $\mathbf{\square}$ : MRI, SPECT and PET.

of the LV myocardium was used to designate the targeted myocardial regions. These data also enabled exact analysis of segmental changes in ventricular function during the follow-up. All patients were screened both before (baseline) and at $1,3,6$, and 12 months after cell transplantations (Fig. 1B).

\section{Diagnostic cardiac imaging}

Cardiac MR imaging was performed using a $1.5-\mathrm{T}$ imager (Siemens $\mathrm{GmbH}$ ). Regional contractility was assessed using a fast, electrocardiography-gated breath-hold steadystate free precession sequence in long axis 2-, 3-, and 4chamber views, as well as LV short axis views. For quantitative analyses, multiple contiguous 10 -mm short-axis slices covering the entire ventricle from apex to base were acquired. Bolus injections of a contrast agent $(0.1 \mathrm{mmol}$ gadoversetamide) were started, and viability imaging was performed beginning after $5 \mathrm{~min}$ by use of phase sensitive inversion recovery segmented gradient echo sequences covering the entire LV myocardium in 2-chamber, 4-chamber, and short-axis planes and repeated until $10 \mathrm{~min}$ after con- trast administration. All MR images were evaluated on a workstation (Synapse 3D, Fujifilm). The right and left ventricular insertion points were defined as reference points for the American Heart Association's (AHA) 16 segment model. Regional wall motion (WM) was evaluated for each segment and classified as normal 1, hypokinesia 2, akinesia 3, and dyskinesia 4. Global LV systolic function parameters were quantified and LVEF was measured. Myocardial wall thickness (WT) was measured manually in the end diastolic phase. Myocardial scar size (SS) was quantified automatically using a dedicated software (Delayed Enhancement, Synapse3D), and was calculated as the percent of myocardial WT in relation to the segment and graded as 1 (0 25\%), 2 (26 50\%), 3 (51 75\%), and $4(76 \sim 100 \%)$. Myocardial mass $(\mathrm{MM})$ was calculated and expressed in grams.

Cardiac Tc-99m-MIBI SPECT imaging was performed using the previously described protocol (18). Myocardial perfusion was applied to patients following $4 \mathrm{~h}$ fasting and $555 \sim 740 \mathrm{MBq}$ of $99 \mathrm{mTc}-\mathrm{MIBI}$ was administered intravenously at rest. Gated Tc-99m-MIBI SPECT was per- 
formed whenever possible. The patients were scanned on dual head SPECT gamma camera (Siemens e-cam) 45 60 min after the injection.

18F-FDG PET imaging was performed as described previously (19) to ensure the uptake by ischemic tissue and optimized suppression of 18-F-FDG uptake by normal myocytes (reduce nonspecific uptake). Patients were imaged on a PET CT scanner (GE Discovery) using a standard protocol after 45 90 min after FDG injection.

The SPECT and PET images were reconstructed and reoriented into standard cardiac planes (short axis, vertical long axis, and horizontal long axis) for interpretation. SPECT and PET imaging were performed with no more than a 1-week interval between each imaging.

\section{Study oversight and monitoring}

A separately constituted data safety and independent monitoring board reviewed the progress of the study, all serious adverse events, and blinded outcomes at 3-month intervals. This board recommended continuation of the trial after each review.

\section{Methods for the assessment of secondary outcomes}

The 6-min walking test, a submaximal exercise test that entails measurement of distance walked over a span of 6 minutes, has been performed due to the American Thoracic Society (ATS) guidelines (20) at designated time points (baseline, 1, 3, 6, and 12 months). A $30 \mathrm{~m}$ distance course (hospital corridor) was used where a person was accompanied the patient. Interpretation of results was guided by functional status of the heart failure and therapeutic monitoring. A single value of the test was reported as an absolute value in meters.

For the assessment of the New York Heart Association (NYHA) functional classification; patients in one of four categories were classified based on their symptoms to address how much they were limited during physical activity at designated time points (baseline, 1, 3, 6, and 12 months). Category I, no limitation of physical activity. Category II, slight limitation of physical activity, comfortable at rest. Category III, marked limitation of physical activity, comfortable at rest. Category IV, unable to carry on any physical activity without discomfort, symptoms of heart failure at rest.

\section{Statistical analysis}

The Shapiro-Wilk test was used to test whether continuous variables were normally distributed. Continuous variables are presented as the mean \pm standard error (SE) unless otherwise stated. One-way analysis of variance
(ANOVA) was performed to compare Echo LVEF values between the groups that were normally distributed. Nonparametric analysis of variance (Kruskal-Wallis) was used to compare continuous variables. When a significant difference was noted, a post-hoc pairwise comparison with a Bonferroni-corrected Mann-Whitney test was performed to analyze the difference between individual groups. The Wilcoxon signed-rank test was performed to compare the baseline and 12-month follow-up data for each group, whereas the paired sample t-test was used to compare myocardial mass. Echo LVEF measurements were analyzed using Friedman non-parametric variance analysis of repeated measurements test (post-hoc Wilcoxon with Bonferroni corrections). Dichotomous variables of groups including smoking, consumption of alcohol or type 2 diabetes were compared using the Chi-square test. Statistical analyses were performed using the IBM SPSS Statistics 22.0 software package (IBM Corp. Released 2013. IBM SPSS Statistics for Windows, Version 22.0. Armonk, NY: IBM Corp.). $\mathrm{p}<0.05$ was considered as an indicator of statistical significance in all analyses. See, Supplementary Table S3 for all statistical tests used to analyze the clinical and the laboratory tests in the HUC-HEART Trial.

\section{Results}

Seventy-three patients were found eligible; 19 were excluded for needing urgent surgery or refusing to participate (Fig. 1A). The remaining 54 patients were randomized in a $1: 1: 2$ pattern and 46 patients completed the 1-year follow-up (Fig. 1B). The patients' demographic data and history are given in Table 1 .

\section{Safety evaluation}

The rate of predefined treatment-related serious adverse events such as anaphylaxis and malignant arrhythmias during follow-up did not occur in any patient. One patient in the control group, 2 patients in the BM-MNC group, and 1 patient in the HUC-MSC group died of low cardiac output within 1 3 months with no direct relation to cell transplantation. No peri-operative myocardial infarction, stroke, infection or delirium was noted. Reactions suggestive of an acute immunogenic reaction such as fever, urticaria, hemolysis, hypotension, liver dysfunction, and/or thrombocytopenia did not occur in any patient following cell transplantations. Twenty-three-parameter laboratory tests showed no significant difference between the groups except the baseline value of the myocardial marker, creatine kinase-muscle/brain (CK-MB), between the control and the BM-MNC groups, albeit within the normal range 
Table 1. Demographics and history of participants who were all Caucasian

\begin{tabular}{|c|c|c|c|c|}
\hline Variables & Control $(n=16)$ & BM-MNC $(n=12)$ & HUC-MSC $(n=25)$ & Significance* \\
\hline Age $($ mean $\pm S E), y r$ & $65.3 \pm 1.7$ & $56.9 \pm 1.5$ & $61.8 \pm 2.0$ & $\chi^{2}=8.049 ; p=0.018^{a}$ \\
\hline $\operatorname{Sex}(M / F)$ & $16 / 0$ & $12 / 0$ & $25 / 0$ & N/A \\
\hline Body mass index $($ mean $\pm \mathrm{SE})$ & $26.6 \pm 1.2$ & $26.2 \pm 0.9$ & $26.5 \pm 0.9$ & $p=0.96$ \\
\hline Smoking $(\mathrm{n} / \%)$ & $15 / 88.2$ & $11 / 91.6$ & $21 / 84.0$ & $\chi^{2}=0.161 ; p=0.923$ \\
\hline Alcohol $(n / \%)$ & 3/17.6 & 2/16.6 & 2/8.0 & $\chi^{2}=0.984 ; p=0.611$ \\
\hline Type II DM (n/\%) & $9 / 52.9$ & $4 / 33.3$ & $16 / 66.7$ & $\chi^{2}=4.376 ; p=0.112$ \\
\hline Hypercholesterolemia & $6 / 42.9$ & $1 / 8.3$ & $10 / 43.5$ & $\chi^{2}=5.083 ; p=0.079$ \\
\hline Hypertension (n/\%) & $11 / 64.7$ & $6 / 50.0$ & $15 / 60.0$ & $\chi^{2}=1.104 ; p=0.576$ \\
\hline TIA (previously) (n/\%) & $1 / 5.9$ & 0/0.0 & 2/8.3 & $\chi^{2}=1.798 ; p=0.407$ \\
\hline Stroke (previously) (n/\%) & $0 / 0$ & $0 / 0$ & $1 / 4.3$ & $\chi^{2}=1.695 ; p=0.429$ \\
\hline No. of bypass grafts (mean \pm SE) & $3.2 \pm 0.2$ & $3.1 \pm 0.3$ & $3.2 \pm 0.2$ & $\chi^{2}=0.134 ; p=0.935$ \\
\hline
\end{tabular}

${ }^{\mathrm{a}}$ The age difference between control and BM-MNC groups were significant $(\mathrm{p}=0.018)$. No significant age difference was noted between control and HUC-MSC groups or BM-MNC and HUC-MSC groups $(p>0.05)$.

T2D: type 2 diabetes, TIA: Transient ischemic attack.

$* \chi^{2}$ : in age, no. of bypass grafts was calculated by Kruskal-Wallis. Others was calculated by Chi-square test.

Table 2. Holter rhythm monitoring (ventricular extrasystoles) at baseline and 12 months after cell transplantation

\begin{tabular}{lcccc}
\multicolumn{1}{c}{ Test time } & Control $(\mathrm{n}=13)$ & BM-MNC $(\mathrm{n}=5)$ & HUC-MSC $(\mathrm{n}=13)$ \\
\hline Baseline & $715.2 \pm 476.2$ & $2,505.2 \pm 1,239.7$ & $859.2 \pm 448.0$ \\
12 months & $673.4 \pm 432.7$ & $1,710.8 \pm 436.8$ & $671.2 \pm 548.3$ & $\mathrm{Z}=1.433 ; \mathrm{p}=0.152$ \\
Time comparison* & $\mathrm{Z}=0.140 ; \mathrm{p}=0.889$ & $\mathrm{Z}=0.405 ; \mathrm{p}=0.686$ & \\
\hline
\end{tabular}

No significant difference was noted between baseline and 12 months follow-up.

All values are given as mean $\pm \mathrm{SE}$.

$*$ Z: Wilcoxon test results.

(Supplementary Table S4). NT-proBNP levels at baseline were comparable between groups $(\mathrm{p}=0.153)$. However, NTproBNP levels between baseline and the follow-up significantly decreased in the BM-MNC group from baseline-month $3(p=0.017), 1 \sim 3$ months $(p=0.043)$, and in the HUC-MSC group from baseline $\sim$ month $3(\mathrm{p}=0.035)$, baseline $\sim$ month $6(\mathrm{p}=0.001)$, month $1 \sim 6(\mathrm{p}=0.001)$, and month $3 \sim 6(\mathrm{p}=0.010)$.

Cardiac supraventricular arrhythmia developed in 1 patient in the BM-MNC group and 1 patient in the HUCMSC group who were successfully controlled with antiarrhythmic treatment in the intensive care unit during $2 \sim 5$ days post-operative. Holter rhythm monitoring values did not differ significantly between baseline and the 12-month follow-up (Table 2). Two extreme results (20,328 and 46,165 ventricular extrasystoles/24 h) were detected in 2 patients in the BM-MNC group, only at month 3, which disappeared afterwards.

\section{Primary outcomes}

During the follow-up, LVEF significantly increased $(\mathrm{p}=$ 0.004) only in the HUC-MSC group (baseline to 12 months) as assessed in MRI (Fig. 2A). LVEF did not significantly change in the control $(p=0.139)$ or BM-MNC groups $(p=$ 0.080) (Fig. 2A). SPECT imaging yielded a significant LVEF increase (baseline to 12 months) both in the BM-MNC $(p=0.015)$ and HUC-MSC $(p=0.044)$ groups, but not in the control group $(\mathrm{p}=0.492)$ (Fig. $2 \mathrm{~B})$. The assessment of LVEF by Echo at consecutive time points showed that there was a significant difference $(p=0.017)$ between baseline and 6 months which was restricted to the HUCMSC group, and not in the BM-MNC $(p=0.075)$ or the control $(p=0.529)$ groups (Fig. 2C). However, cumulative analyses of LVEF change on patient-based analysis using MRI, SPECT, and Echo revealed no significance between the groups $(\mathrm{p}=0.376 ; \mathrm{p}=0.110$, and $\mathrm{p}=0.765$, respectively) (Fig. 2D).

The AHA's 16-segment model was mapped using MRI to assess the LV remodeling by WT, SS, WM, and MM. Segmental healing/worsening (as assessed by four parameters, see Materials and Methods, Diagnostic Cardiac Imaging) between baseline and 12-months assessed by MRI, was one of the primary outcomes of this trial, as represented in Fig. 3 and Fig. 4. Any positively-changed ven- 


\section{A. LVEF change measured by MRI}

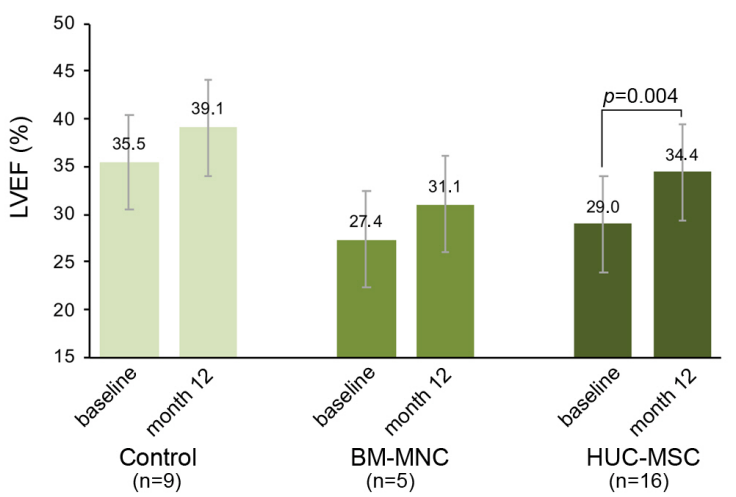

C. LVEF change measured by Echo

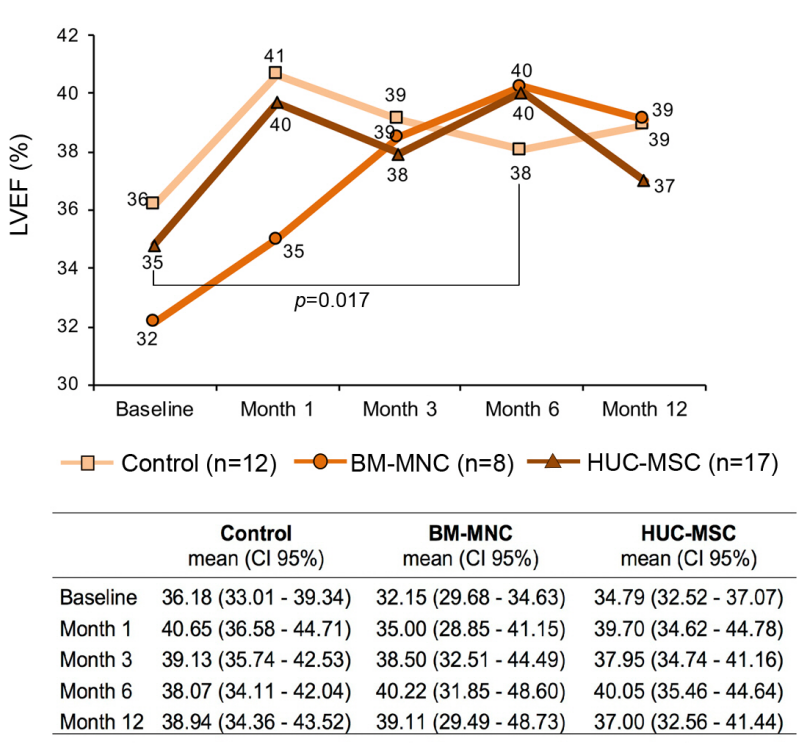

\section{B. LVEF change measured by SPECT}

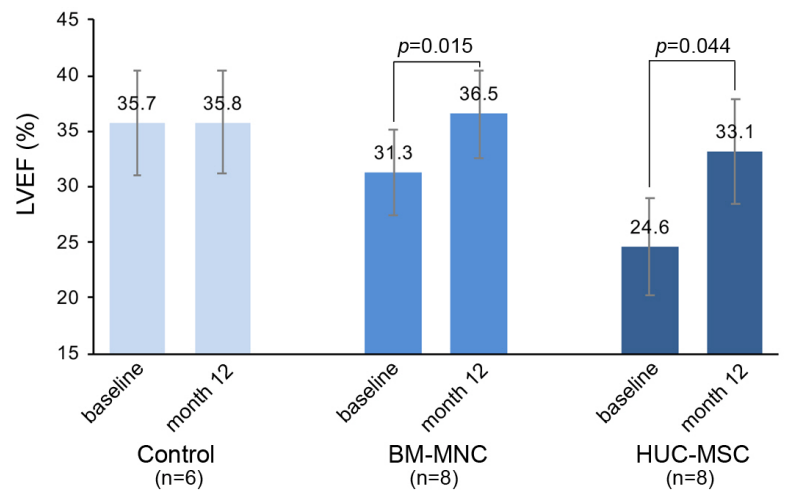

D. LVEF change ratio measured by MRI, SPECT and Echo

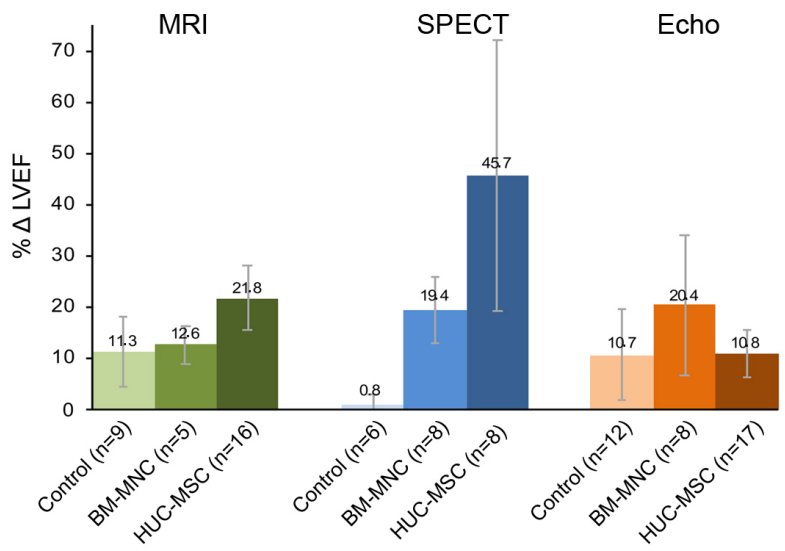

Fig. 2. Changes in LVEF (\%) measured by MRI (A); SPECT (B); Echo (C), and calculated LVEF change ratio ( $\Delta$ LVEF) at baseline and after 12-month follow-up (D). (A) LVEF significantly increased only in HUC-MSC group $(p=0.004)$; did not significantly change in the control or BM-MNC groups (see, text for $p$ values). (B) SPECT imaging presented significant LVEF increase both in BM-MNC ( $p=0.015)$ and HUC-MSC ( $p=0.044$ ) groups. (C) LVEF (baseline to 6 month) detected using Echo, and was also found different only in HUC-MSC group $(p=0.017)$. Mean LVEF $(\%)$ and confidence intervals $(\mathrm{Cl} 95 \%)$ at certain time-points are given in the table below. (D) Cumulative LVEF change ratio in each patient $(\triangle \mathrm{LVEF})$ in MRI, SPECT, and Echo revealed no significance between groups $(p=0.376 ; p=0.110$, and $p=0.765$, respectively).

tricular parameter was considered as a healing response. Based on the results of segmental analysis, responder vs nonresponder ratio was calculated and expressed in Fig. $3 \mathrm{~A}$ and $3 \mathrm{~B}$ corresponding to the wall motion and scar scores, respectively. The responder ratio was found higher in all groups compared with nonresponders regarding these two ventricular parameters (green bars vs red bars in Fig. $3 \mathrm{~A}$ and $3 \mathrm{~B})$. The response ratio was strikingly higher in the HUC-MSC group ( $94 \%$ and $87 \%$ in WM and SS, re- spectively). The WT change in the control and the cell treatment groups revealed gradual increases among the groups (Fig. 3C). In the HUC-MSC group, a $9.2 \pm 2.0 \mathrm{~mm}$ increase and $11.5 \pm 2.0 \mathrm{~mm}$ decrease was identified. However, no significant difference was found between the groups $\left(\chi^{2}=2.400 ; \mathrm{p}=0.301\right)$.

Finally, MM changes were analyzed using MRI data. MM change in grams was not different within each group $(p>0.05)$ or between groups $(p=0.621)$. However, it is 
A. Percentage of response in wall motion (WM)

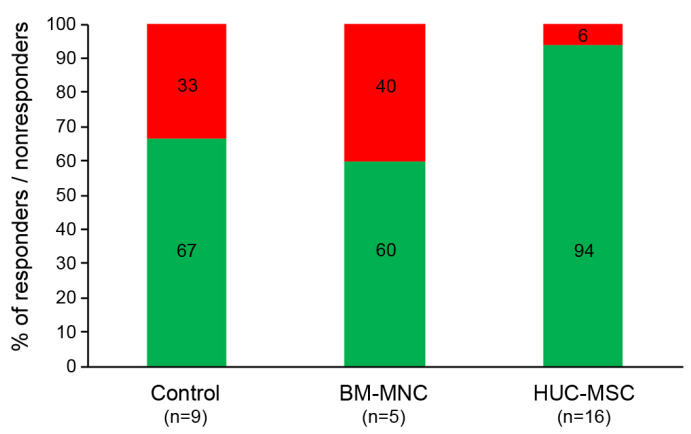

C. Wall thickness (WT) change

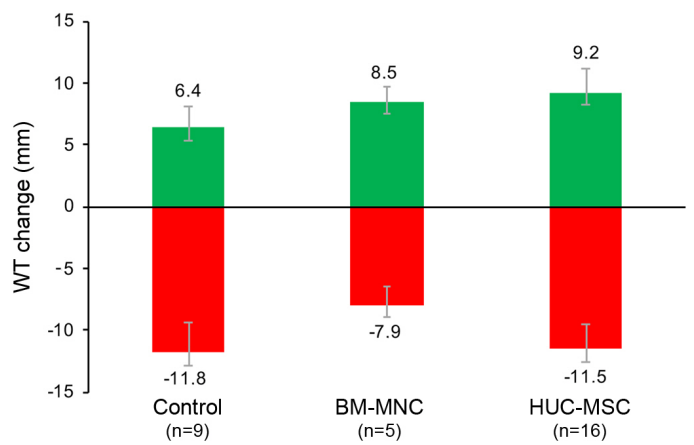

B. Percentage of response in scar score (SS)

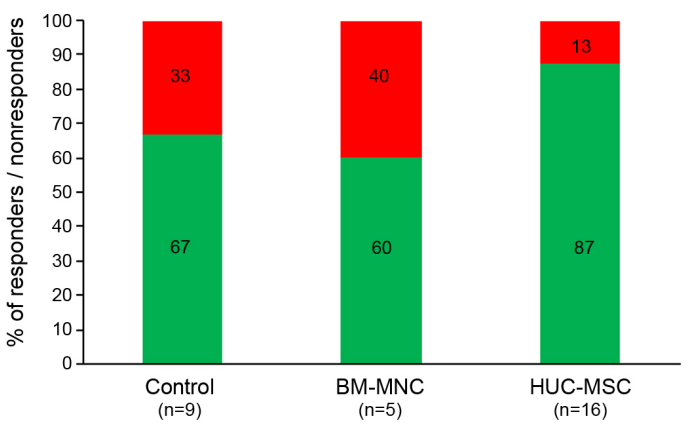

D. Myocardial mass (MM) change

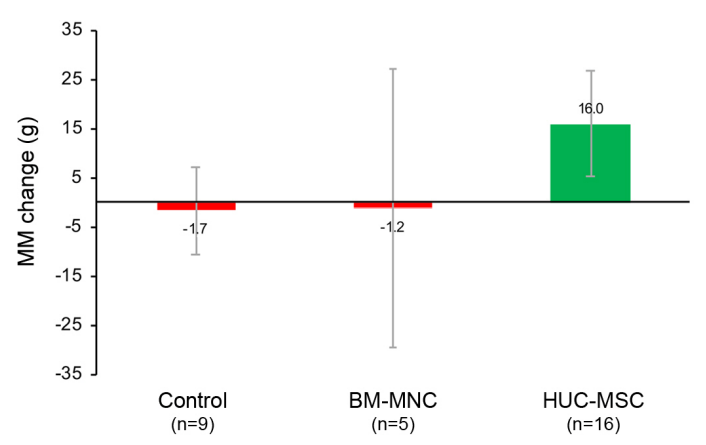

Fig. 3. Ratio of responder vs nonresponder and left ventricle measurements based on the segmental MRI evaluations. (A, B) represent the ratio of responder (green bars) vs nonresponder (red bars) due to the healing of wall motion (WM) and scar score (SS), respectively. (C) Wall thickness $(\mathrm{WT})$ change $(\mathrm{mm})$ showed no significant difference between the groups $\left(\chi^{2}=2.400 ; p=0.301\right)$. (D) Myocardial mass (MM) change (grams) between the baseline and after the 12-month follow-up was not different within each group $(p=0.850)$ or between groups $(p=0.440)$. However, a striking MM increase $(16 \pm 10.6 \mathrm{~g})$ was noted only in HUC-MSC group, whereas MM decreased in the control and BM-MNC groups. Although none of the measured/calculated values based on the segmental analysis (wall motion, scar score, wall thickness and myocardial mass) exhibited significant difference among groups, these results demonstrated that a higher degree of healing effect in the HUC-MSC group (compare the values in the A D).

A. Hibernating area measured by PET

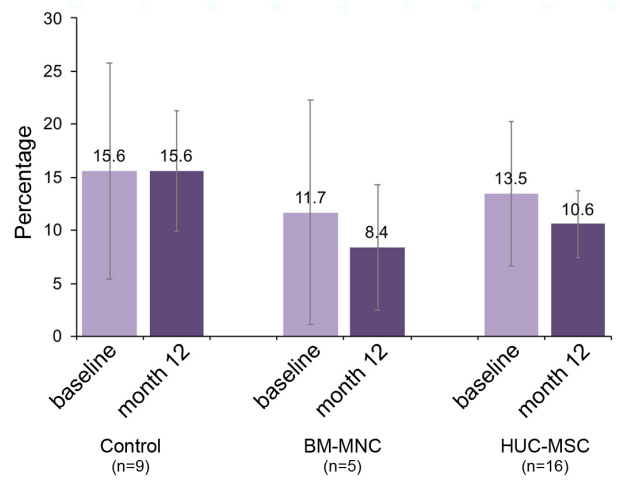

B. Necrotic area measured by PET

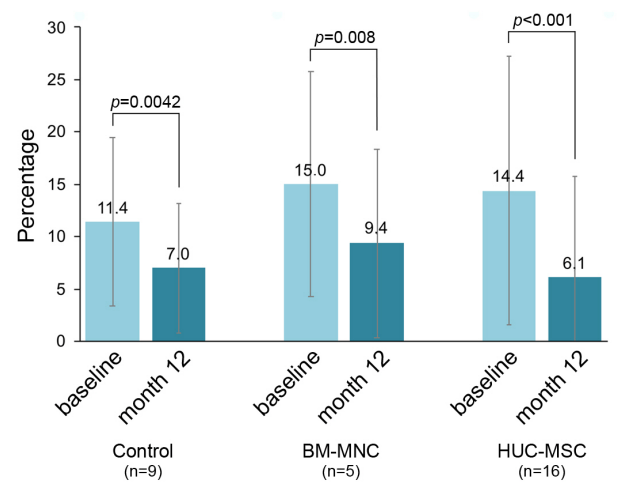

Fig. 4. Changes (percentage) in hibernating (A) and necrotic area (B) measured by PET. Cell-treated groups demonstrated a decrease in hibernating area; no significance difference was noted among groups or within each group. Varying and significant decreases were noted in the necrotic myocardium (baseline-12-month) as $2.3 \%$ in the control $(Z=2.033 ; p=0.042), 4.5 \%$ in $B M-M N C(Z=2.666 ; p=0.008)$, and $7.7 \%$ in the HUC-MSC $(Z=3.517 ; p<0.001)$ groups. Graphics were drawn using median and error bars were given as IQR/2. 


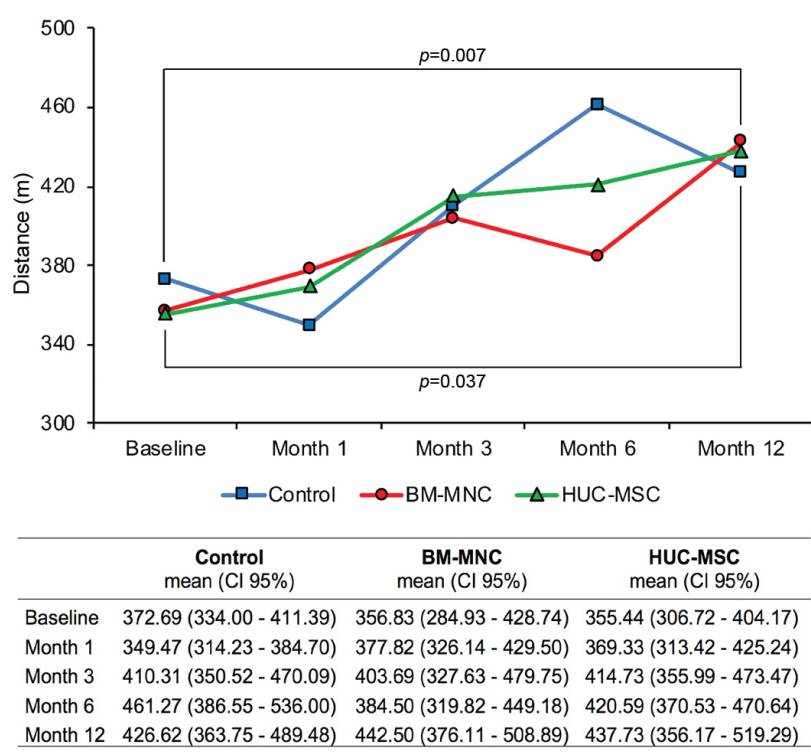

Fig. 5. Changes in 6-min walking distance (in meters) throughout the 12-month follow-up. Significant increase was noticed in the control $(p=0.007)$ and HUC-MSC $(p=0.037)$ groups only between baseline and at the end of the 12-month distances. Mean walked distances in meters and confidence intervals $(\mathrm{Cl} 95 \%)$ at certain time points are given in the table below.

worthwhile mentioning that a remarkable $\mathrm{MM}$ increase $(16 \pm 10.6 \mathrm{~g})$ was noticed only in the HUC-MSC group, but a decline in the control and BM-MNC groups (Fig. 3D).

A series of further measurable parameters listed in Supplementary Table S5 demonstrated that stroke volume (SV) increased significantly $(19.7 \%)(\mathrm{Z}=2.637 ; \mathrm{p}=0.008)$ only in HUC-MSC group at month 12 compared with baseline.

Hibernating and necrotic myocardium was assessed using PET scans at baseline and at the end of the follow-up. Only cell-treated groups demonstrated a decrease in hibernating areas; however, no significant difference was noted among the groups or within each group (Fig. 4A). Necrotic area calculations revealed varying degrees of decline (baseline and 12 months) in all groups as $2.3 \%$ in the controls $(\mathrm{Z}=2.033 ; \mathrm{p}=0.042), 4.5 \%$ in the $\mathrm{BM}-\mathrm{MNC}(\mathrm{Z}=2.666 ; \mathrm{p}=$ $0.008)$ and $7.7 \%$ in the HUC-MSC $(Z=3.517 ; p<0.001)$ groups (Fig. 4B). Necrotic area decline was noted in 7/9, 9/9, and 16/16 patients in the control, BM-MNC, and HUCMSC groups, respectively. One patient remained stable, and one patient worsened in the control group.

\section{Secondary (functional) outcomes}

The 6-min walk test revealed a significant increase in the control $(14.5 \% ; \mathrm{p}=0.007)$ and HUC-MSC $(13.8 \% ; \mathrm{p}=$ 0.037) groups (baseline to 12 months) (Fig. 5). The mean
Table 3. Functional capacity by NYHA grading at baseline and at the end of 12-month follow-up

\begin{tabular}{lcccc}
\hline & $\begin{array}{c}\text { Baseline } \\
\text { mean }( \pm \text { SE) }\end{array}$ & $\begin{array}{c}\text { Improved } \\
n,(\%)\end{array}$ & $\begin{array}{c}\text { Same } \\
n,(\%)\end{array}$ & $\begin{array}{c}\text { Worsened } \\
n,(\%)\end{array}$ \\
\hline Control $(\mathrm{n}=14)$ & $2.1 \pm 0.1$ & $7,(50)$ & $6,(42)$ & $1,(7)$ \\
BM-MNC $(\mathrm{n}=9)$ & $2.2 \pm 0.2$ & $6,(66)$ & $3,(33)$ & $0,(0)$ \\
HUC-MSC $(\mathrm{n}=19)$ & $1.9 \pm 0.1$ & $8,(42)$ & $10,(52)$ & $1,(5)$ \\
\hline
\end{tabular}

No patient has been graded in NYHA IV category. No significant difference was noted between groups, $\mathrm{p}=0.338$.

distance change in the control group was $76.9 \pm 23.0 \mathrm{~m}(\mathrm{p}=$ $0.007), 91.1 \pm 63.1 \mathrm{~m}(\mathrm{p}=0.199)$ in the BM-MNC group, and $102.5 \pm 43.6 \mathrm{~m}(\mathrm{p}=0.037)$ in the HUC-MSC group.

The New York Heart Association (NYHA) scores improved at the end of follow-up compared with baseline in 7 patients (50\%) in the control, 6 patients (66\%) in the BM-MNC, and 8 patients (42\%) in the HUC-MSC group (Table 3). No significant difference was noted between the groups at baseline $(\mathrm{p}=0.338)$.

\section{Discussion}

The HUC-HEART Trial was designed to assess the safety and efficacy of allogeneic HUC-MSCs through a comparison with no cell implanted controls and patients who underwent autologous BM-MNC-transplantation. To the best of our knowledge, this is the first study in the literature in two aspects; (i) the efficacy of HUC-MSCs as examined upon direct administration into the myocardium; (ii) the efficacy of BM-MNC injections was compared side-by-side in the same clinical settings. The primary outcomes were determined as the evaluation of LVEF using Echo and more advanced imaging techniques including cardiac MRI and SPECT. As being the key point in cell-based therapies, the degree of myocardial remodeling was also ascertained using MRI-based segmental measurements and scorings (WM, SS, WT and MM). Here, we report a detailed, 16-segmental analysis, rather than just assessing the global recovery of the ventricular functions because cardiac MRI is considered the gold standard for the assessment of fibrosis/scar and is commonly used in the evaluation of patients with significant coronary artery disease and cardiomyopathy prior to formulating a treatment strategy (21). Further evaluation of functional cardiomyocyte metabolism with PET (22), provided invaluable data on the status of hibernating and necrotic myocardium in this trial. Besides the significant increase in LVEF restricted to the cell therapy groups, a more important finding was the improvement in the necrotic areas where sig- 
nificant declines were noted. However, no statistical difference $(p=0.123)$ was calculated in the mean differences between baseline and 12-month among the groups. Nevertheless, it is noteworthy to mention that the highest values were obtained in the HUC-MSC group. According to the LVEF Echo results as illustrated in Fig. 2C, the control and BM-MNC group showed no statistically significance starting from baseline to 12 months. Further dissecting the values measured at certain time points, the slight drop in BM-MNC and HUC-MSC groups after 6 months also presented no significance. Among the three groups, only the HUC-MSC group exhibited a significant increase in the LVEF between baseline and the 6-month follow-up.

One of the primary outcomes of this trial was the MRI-based assessment of four parameters (WM, SS, WT and $\mathrm{MM}$ ), all of which directly reflect that the ventricular functions were expressed as segmental healings/worsening. Any positivity was considered as "a response" to the intervention, either in CABG alone group (control) or celltreatment groups because all patients had an infarcted necrotic myocardium (single or multiple) at baseline. In other words, because the healing of a macroscopic necrotic tissue in the heart does not normally occur if no treatment is administered (1), such parameters do not notably chance, when an intervention has not been applied. As such, any positively changed parameter in this trial was considered as a healing response. Undoubtedly, a 100\% response in the cell therapy groups would correspond to significant elevations in all parameters. We did not note any patient displaying $100 \%$ response upon CABG + cell administration.

Another supportive finding was the MM increase, which was restricted to HUC-MSC group. It is obvious that CABG surgery per se may provide myocardial restoration (23). However, given that all patients were subjected to identical conditions including CABG surgery, the significant differences between the groups and/or within each group demonstrated the improvement of clinical endpoints. Segmental recoveries give further credence to the fact that myocardial integration of transplanted MSCs (24) was achieved to some extent and exhibited long-lasting paracrine effects.

MRI measurements and calculations related to the ventricular volumes showed a significant increase only in SV, which was exclusive to the HUC-MSC group, although lesser degree of increases was also noted in the control and BM-MNC groups (close to the limit of significance). This situation may directly correspond to the segmental healing of the ventricle, particularly in the HUC-MSC group. However, the SV increase did not reach to the global heal- ing level, possibly because of the insignificant increases of cardiac output and cardiac index, and therefore stayed in the shadow of other parameters.

Although safety is still considered an important issue in cell therapies regarding no-reflow after intracardiac cell injections; in the past two decades, several clinical trials with adult stem cells of different tissue origins administered for myocardial restoration report no major adverse safety issues (2). More specifically, trials using HUC-MSCs in patients with heart failure reported no serious and longterm clinical adverse effects $(10-13,15,16,25)$. We also encountered no short- or mid-term adverse events including malignant ventricular arrhythmia, implying that HUCMSCs are not harmful, at least at the tested doses. NTproBNP measurements simply indicated that the BM-MNC and HUC-MSC groups had no detrimental effect on ventricular functions. Moreover, the NT-proBNP levels in both cell-treated groups indicated a noteworthy healing effect compared with the control group, especially during the first 6 months of follow-up.

To date, HUC-MSCs have been administered only in seven published clinical trials for the treatment of acute or chronic cardiac ischemia or heart failure $(10-13,15,16$, $25)$. Based on those seven trials, the cell dose administered varied between $3 \times 10^{6} \sim 70 \times 10^{6}$, so the mean number was around $20 \times 10^{6}$ cells per patient. Cells were not administered by the intramyocardial route in any of those trials (four intracoronary; two intravenous and one trans-coronary). Obviously, injecting a high volume of cells to the peri-infarct myocardium (ischemic area was usually around $1 \sim 2 \mathrm{~cm}^{2}$ ) was not feasible. Unlike BM-MNCs, MSCs have substantially larger cell size; therefore, the number of cells in a diluting media higher than a certain amount may result in cell clogging during storage and injection. Thus, we set the final cell concentration to $2.1 \sim 2.6 \times 10^{6}$ cells per $400 \mu 1$ diluent to inject a total of $21 \sim 26 \times 10^{6}$ cells divided into approximately ten peri-infarct sites in a total of $4 \mathrm{ml}$ diluent. This trial provides no data related to the comparison of varying cell doses.

Preclinical studies have demonstrated that HUC-MSCs are superior in expressing structural cardiomyocytic molecules such as troponin-I, connexin-43; thus differentiating into cardiomyocyte and endothelial cells in vitro, (26) also exerting paracrine effects that enhance vascular regeneration, neovascularization and cardiomyocyte protection, and reducing myocardial fibrosis and apoptosis $(27,28)$. The incorporation of MSCs into tissues is regulated by multiple processes, including cell recruitment, migration, and adhesion (29). Bartolucci et al. (10) demonstrated that HUC-MSCs had a putative higher tissue-repair paracrine 
potential compared with other adult tissue-derived MSCs and also possessed a greater migration capacity in response to patient serum with heart failure, a finding which may indicate that HUC-MSCs sense biologic cues that are contributory to their therapeutic effect by systemic and/or local deliveries. Nevertheless, route of delivery of MSCs does matters as intramyocardial injections rather than intracoronary infusions had a more profound effect on LVEF (30, 31). It was suggested that BM-MSCs injected directly into the cardiac muscle or in the periphery of the infarct may be retained more efficiently than cells infused via the coronary arteries (31). Cells infused into the arteries find the target through their limited capacity to migrate towards the injured area, whereas by delivering cells directly into the cardiac muscle and/or in the vicinity of the scar area, the hurdle of recruiting cells to the injured tissue would partially be overcome. Nevertheless, a well-designed comparative study of intracoronary versus intramyocardial HUC-MSC administration is still necessary as the catheter-based intracoronary intervention is obviously less invasive technique than open-chest surgerybased intramyocardial delivery.

The HUC-MSC trials published so far are still at phase I or II, and safety was the primary endpoint in almost all studies. Although it is too soon to confirm the therapeutic efficacy of these early-phase small-scale clinical trials, controlled studies including ours demonstrate the solid improvement of LVEF in HUC-MSC transplantations compared with control groups. In addition to the improvement in global LV function, prevention of adverse cardiac remodeling in patients with acute myocardial ischemia who received intracoronary infusion of HUC-MSCs has also been reported (13). Significant improvements were also noted in exercise tolerance and clinical status after HUCMSC treatment (11) as well as an increase in 6-min walking distances in the present study and by others (11) associated with decreases in serum NT-proBNP levels, a finding that was identified in BM-MNC- and more strikingly in the HUC-MSC-receiving patients.

In this phase I/II clinical trial, intramyocardial administration of HUC-MSCs in combination with CABG displayed higher scores in reducing the scar tissue and restoration of ventricular wall functions compared with autologous BM-MNCs; thus, HUC-MSCs may have a therapeutic potential for patients with CIC as directly administered cells seem to integrate to the recipient myocardium and exhibited the well-established paracrine functions (overall significant results are presented in a graphical abstract). Further larger scale, phase III clinical trials are indispensable. Additionally, establishment of appropriate facilities to produce large-scale, off-the-shelf HUC-MSCs seems mandatory and feasible for further testing the therapeutic use of these cells in cardiomyopathic disorders.

\section{Limitations}

This study has the following limitations, mostly due to technical restrictions that occurred during patient enrollment, interventions and/or follow-up: (i) Due to ethical concerns, control patients did not receive any placebo myocardial injections. (ii) Due to feasibility reasons, cells had to be injected in two different vehicle volumes $(10 \mathrm{ml}$ vs. $4 \mathrm{ml}$ ) for the two groups. (iii) Only male patients were enrolled in the study. Though, eliminating the confounder factor arising from the sex variation yielded more accurate results in this relatively small-scale trial. (iv) Due to budget limitations as allocated for a given period, only a limited number of patients were enrolled using pre-specified strict inclusion/exclusion criteria. (v) Some enrolled patients either missed PET scanning due to blood sugar maladaptation during imaging or failed to undergo MRI during follow-up because of implantable cardioverter-defibrillator (ICD) implantation.

\section{Acknowledgments}

This study was funded in part by The Ministry of Industry and Technology (0741-STZ-2014) (75\%), ATIGENCELL Technologies Cell and Gene Center (15\%), and University Research Fund (17A0230001) (10\%). The authors have no conflicts of interest to declare regarding the publication of the data and the manuscript.

\section{Potential Conflict of Interest}

The authors have no conflicting financial interest.

\section{Supplementary Materials}

Supplementary data including five tables can be found with this article online at http://pdf.medrang.co.kr/paper/ pdf/IJSC/IJSC-12-s20075.pdf.

\section{References}

1. Cahill TJ, Choudhury RP, Riley PR. Heart regeneration and repair after myocardial infarction: translational opportunities for novel therapeutics. Nat Rev Drug Discov 2017; 16:699-717

2. Ghiroldi A, Piccoli M, Cirillo F, Monasky MM, Ciconte G, Pappone C, Anastasia L. Cell-based therapies for cardiac regeneration: a comprehensive review of past and ongoing strategies. Int J Mol Sci 2018;19:3194

3. Arnous S, Mozid A, Martin J, Mathur A. Bone marrow 
mononuclear cells and acute myocardial infarction. Stem Cell Res Ther 2012;3:2

4. Colicchia M, Jones DA, Beirne AM, Hussain M, Weeraman D, Rathod K, Veerapen J, Lowdell M, Mathur A. Umbilical cord-derived mesenchymal stromal cells in cardiovascular disease: review of preclinical and clinical data. Cytotherapy 2019;21:1007-1018

5. Fisher SA, Brunskill SJ, Doree C, Mathur A, Taggart DP, Martin-Rendon E. Stem cell therapy for chronic ischaemic heart disease and congestive heart failure. Cochrane Database Syst Rev 2014;(4):CD007888

6. Clifford DM, Fisher SA, Brunskill SJ, Doree C, Mathur A, Watt S, Martin-Rendon E. Stem cell treatment for acute myocardial infarction. Cochrane Database Syst Rev 2012; (2):CD006536

7. Can A, Yigman Z. Mesenchymal stem/stromal cells from neonatal tissues. In: Bolontrade MF, Garcia MG, editor. Mesenchymal stromal cells as tumor stromal modulators. London: Academic Press; 2017. 65-101

8. Can A, Celikkan FT, Cinar O. Umbilical cord mesenchymal stromal cell transplantations: a systemic analysis of clinical trials. Cytotherapy 2017;19:1351-1382

9. Can A, Ulus A, Topal Celikkan F, Mungan C, Sucu M, Kurtoglu M, Simsek E, Cinar O. Preliminary results of the human umbilical cord mesenchymal stromal cell (HUCMSC) transplantation in myocardial ischemia (HUC-HEART Trial). Paper presented at: International Society for Stem Cell Research (ISSCR) Annual Meeting; 2017 June 14-17; Boston, MA, USA

10. Bartolucci J, Verdugo FJ, González PL, Larrea RE, Abarzua E, Goset C, Rojo P, Palma I, Lamich R, Pedreros PA, Valdivia G, Lopez VM, Nazzal C, Alcayaga-Miranda F, Cuenca J, Brobeck MJ, Patel AN, Figueroa FE, Khoury $M$. Safety and efficacy of the intravenous infusion of umbilical cord mesenchymal stem cells in patients with heart failure: a phase $1 / 2$ randomized controlled trial (RIMECARD Trial [Randomized Clinical Trial of Intravenous Infusion Umbilical Cord Mesenchymal Stem Cells on Cardiopathy]). Circ Res 2017;121:1192-1204

11. Zhao XF, Xu Y, Zhu ZY, Gao CY, Shi YN. Clinical observation of umbilical cord mesenchymal stem cell treatment of severe systolic heart failure. Genet Mol Res 2015;14: 3010-3017

12. Musialek P, Mazurek A, Jarocha D, Tekieli L, Szot W, Kostkiewicz M, Banys RP, Urbanczyk M, Kadzielski A, Trystula M, Kijowski J, Zmudka K, Podolec P, Majka M. Myocardial regeneration strategy using Wharton's jelly mesenchymal stem cells as an off-the-shelf 'unlimited' therapeutic agent: results from the acute myocardial infarction first-in-man study. Postepy Kardiol Interwencyjnej 2015;11: 100-107

13. Gao LR, Chen Y, Zhang NK, Yang XL, Liu HL, Wang ZG, Yan XY, Wang Y, Zhu ZM, Li TC, Wang LH, Chen HY, Chen YD, Huang CL, Qu P, Yao C, Wang B, Chen GH, Wang ZM, Xu ZY, Bai J, Lu D, Shen YH, Guo F, Liu MY, Yang Y, Ding YC, Yang Y, Tian HT, Ding QA, Li LN,
Yang XC, Hu X. Intracoronary infusion of Wharton's jelly-derived mesenchymal stem cells in acute myocardial infarction: double-blind, randomized controlled trial. BMC Med 2015;13:162

14. Can A, Ulus AT, Cinar O, Topal Celikkan F, Simsek E, Akyol M, Canpolat U, Erturk M, Kara F, Ilhan O. Human umbilical cord mesenchymal stromal cell transplantation in myocardial ischemia (HUC-HEART Trial). A study protocol of a phase $1 / 2$, controlled and randomized trial in combination with coronary artery bypass grafting. Stem Cell Rev Rep 2015;11:752-760

15. Bilal M, Haseeb A, Sher Khan MA. Intracoronary infusion of Wharton's jelly-derived mesenchymal stem cells: a novel treatment in patients of acute myocardial infarction. J Pak Med Assoc 2015;65:1369

16. Li X, Hu YD, Guo Y, Chen Y, Guo DX, Zhou HL, Zhang FL, Zhao QN. Safety and efficacy of intracoronary human umbilical cord-derived mesenchymal stem cell treatment for very old patients with coronary chronic total occlusion. Curr Pharm Des 2015;21:1426-1432

17. Celikkan FT, Mungan C, Sucu M, Ulus AT, Cinar O, Ili EG, Can A. Optimizing the transport and storage conditions of current Good Manufacturing Practice-grade human umbilical cord mesenchymal stromal cells for transplantation (HUC-HEART Trial). Cytotherapy 2019;21:6475

18. Holly TA, Abbott BG, Al-Mallah M, Calnon DA, Cohen MC, DiFilippo FP, Ficaro EP, Freeman MR, Hendel RC, Jain D, Leonard SM, Nichols KJ, Polk DM, Soman P. Single photon-emission computed tomography. J Nucl Cardiol 2010;17:941-973

19. Dilsizian V, Bacharach SL, Beanlands RS, Bergmann SR, Delbeke D, Dorbala S, Gropler RJ, Knuuti J, Schelbert HR, Travin MI. ASNC imaging guidelines/SNMMI procedure standard for positron emission tomography (PET) nuclear cardiology procedures. J Nucl Cardiol 2016;23:1187-1226

20. Guyatt GH, Sullivan MJ, Thompson PJ, Fallen EL, Pugsley SO, Taylor DW, Berman LB. The 6-minute walk: a new measure of exercise capacity in patients with chronic heart failure. Can Med Assoc J 1985;132:919-923

21. Kancharla K, Weissman G, Elagha AA, Kancherla K, Samineni S, Hill PC, Boyce S, Fuisz AR. Scar quantification by cardiovascular magnetic resonance as an independent predictor of long-term survival in patients with ischemic heart failure treated by coronary artery bypass graft surgery. J Cardiovasc Magn Reson 2016;18:45

22. Obrzut S, Jamshidi N, Karimi A, Birgersdotter-Green U, Hoh C. Imaging and modeling of myocardial metabolism. J Cardiovasc Transl Res 2010;3:384-396

23. Michler RE, Rouleau JL, Al-Khalidi HR, Bonow RO, Pellikka PA, Pohost GM, Holly TA, Oh JK, Dagenais F, Milano C, Wrobel K, Pirk J, Ali IS, Jones RH, Velazquez EJ, Lee KL, Di Donato M. Insights from the STICH trial: change in left ventricular size after coronary artery bypass grafting with and without surgical ventricular reconstruction. J Thorac Cardiovasc Surg 2013;146:1139-1145.e6 
24. Karantalis V, DiFede DL, Gerstenblith G, Pham S, Symes J, Zambrano JP, Fishman J, Pattany P, McNiece I, Conte J, Schulman S, Wu K, Shah A, Breton E, Davis-Sproul J, Schwarz R, Feigenbaum G, Mushtaq M, Suncion VY, Lardo AC, Borrello I, Mendizabal A, Karas TZ, Byrnes J, Lowery M, Heldman AW, Hare JM. Autologous mesenchymal stem cells produce concordant improvements in regional function, tissue perfusion, and fibrotic burden when administered to patients undergoing coronary artery bypass grafting: The Prospective Randomized Study of Mesenchymal Stem Cell Therapy in Patients Undergoing Cardiac Surgery (PROMETHEUS) trial. Circ Res 2014;114:1302-1310.

25. Fang Z, Yin X, Wang J, Tian N, Ao Q, Gu Y, Liu Y. Functional characterization of human umbilical cord-derived mesenchymal stem cells for treatment of systolic heart failure. Exp Ther Med 2016;12:3328-3332

26. Ramkisoensing AA, Pijnappels DA, Askar SF, Passier R, Swildens J, Goumans MJ, Schutte CI, de Vries AA, Scherjon S, Mummery CL, Schalij MJ, Atsma DE. Human embryonic and fetal mesenchymal stem cells differentiate toward three different cardiac lineages in contrast to their adult counterparts. PLoS One 2011;6:e24164

27. Liu CB, Huang H, Sun P, Ma SZ, Liu AH, Xue J, Fu JH, Liang YQ, Liu B, Wu DY, Lü SH, Zhang XZ. Human umbilical cord-derived mesenchymal stromal cells improve left ventricular function, perfusion, and remodeling in a por- cine model of chronic myocardial ischemia. Stem Cells Transl Med 2016;5:1004-1013

28. Santos Nascimento D, Mosqueira D, Sousa LM, Teixeira M, Filipe M, Resende TP, Araújo AF, Valente M, Almeida J, Martins JP, Santos JM, Bárcia RN, Cruz P, Cruz H, Pinto-do-Ó P. Human umbilical cord tissue-derived mesenchymal stromal cells attenuate remodeling after myocardial infarction by proangiogenic, antiapoptotic, and endogenous cell-activation mechanisms. Stem Cell Res Ther 2014;5:5

29. Huang J, Zhang Z, Guo J, Ni A, Deb A, Zhang L, Mirotsou M, Pratt RE, Dzau VJ. Genetic modification of mesenchymal stem cells overexpressing CCR1 increases cell viability, migration, engraftment, and capillary density in the injured myocardium. Circ Res 2010;106:1753-1762

30. Brunskill SJ, Hyde CJ, Doree CJ, Watt SM, Martin-Rendon E. Route of delivery and baseline left ventricular ejection fraction, key factors of bone-marrow-derived cell therapy for ischaemic heart disease. Eur J Heart Fail 2009;11:887896

31. Ang KL, Chin D, Leyva F, Foley P, Kubal C, Chalil S, Srinivasan L, Bernhardt L, Stevens S, Shenje LT, Galiñanes M. Randomized, controlled trial of intramuscular or intracoronary injection of autologous bone marrow cells into scarred myocardium during CABG versus CABG alone. Nat Clin Pract Cardiovasc Med 2008;5:663-670 